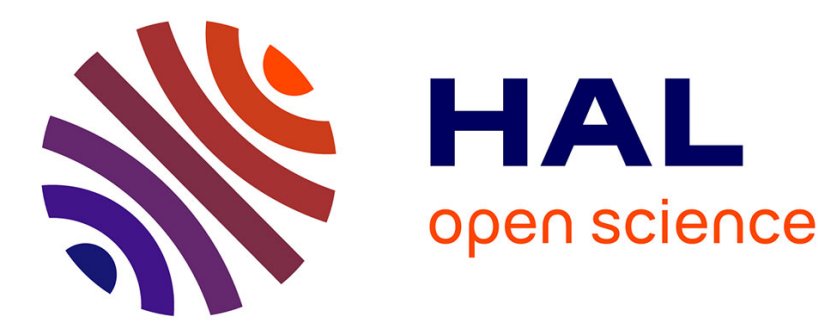

\title{
Electromagnetic wave propagation in quasiperiodic photonic circuits
}

\author{
E.H. El Boudouti, Y. El Hassouani, H. Aynaou, Bahram Djafari-Rouhani,
} Abdellatif Akjouj, V.R. Velasco

\section{- To cite this version:}

E.H. El Boudouti, Y. El Hassouani, H. Aynaou, Bahram Djafari-Rouhani, Abdellatif Akjouj, et al.. Electromagnetic wave propagation in quasiperiodic photonic circuits. Journal of Physics: Condensed Matter, 2007, 19, pp.246217/1-20. 10.1088/0953-8984/19/24/246217 . hal-00283126

\section{HAL Id: hal-00283126 \\ https://hal.science/hal-00283126}

Submitted on 26 Aug 2021

HAL is a multi-disciplinary open access archive for the deposit and dissemination of scientific research documents, whether they are published or not. The documents may come from teaching and research institutions in France or abroad, or from public or private research centers.
L'archive ouverte pluridisciplinaire HAL, est destinée au dépôt et à la diffusion de documents scientifiques de niveau recherche, publiés ou non, émanant des établissements d'enseignement et de recherche français ou étrangers, des laboratoires publics ou privés.

\section{(c)(1)}

Distributed under a Creative Commons Attribution| 4.0 International License 


\title{
Electromagnetic wave propagation in quasi-periodic photonic circuits
}

\author{
E H El Boudouti ${ }^{1}$, Y El Hassouani ${ }^{1}$, H $_{\text {Aynaou }}{ }^{1}$, B Djafari-Rouhani ${ }^{2}$, \\ A Akjouj ${ }^{2}$ and V R Velasco ${ }^{3}$ \\ ${ }^{1}$ Laboratoire de Dynamique et d'Optique des Matériaux, Département de Physique, Faculté des \\ Sciences, Université Mohamed Premier, 60000 Oujda, Morocco \\ ${ }^{2}$ Institut d'électronique, de Microélectronique et de Nanotechnologie (IEMN), UMR CNRS 8520, \\ UFR de Physique, Université de Lille 1, 59655 Villeneuve d'Ascq, France \\ ${ }^{3}$ Instituto de Ciencia de Materiales de Madrid, CSIC, Sor Juana Inés de la Cruz 3, 28049 Madrid, \\ Spain
}

E-mail: elboudouti@yahoo.fr

\begin{abstract}
We study theoretically and experimentally the properties of quasiperiodic one-dimensional serial loop structures made of segments and loops arranged according to a Fibonacci sequence (FS). Two systems are considered. (i) By inserting the FS horizontally between two waveguides, we give experimental evidence of the scaling behaviour of the amplitude and the phase of the transmission coefficient. (ii) By grafting the FS vertically along a guide, we obtain from the maxima of the transmission coefficient the eigenmodes of the finite structure (assuming the vanishing of the magnetic field at the boundaries of the FS). We show that these two systems (i) and (ii) exhibit the property of self-similarity of order three at certain frequencies where the quasiperiodicity is most effective. In addition, because of the different boundary conditions imposed on the ends of the FS, we show that horizontal and vertical structures give different information on the localization of the different modes inside the FS. Finally, we show that the eigenmodes of the finite FS coincide exactly with the surface modes of two semi-infinite superlattices obtained by the cleavage of an infinite superlattice formed by a periodic repetition of a given FS.
\end{abstract}

\section{Introduction}

Photonic crystals (PCs) have been a subject of great interest during the last decade because of their interesting properties in the development of new optical circuits [1,2]. These systems, constituted by periodic arrangements (cells) of dielectric materials according to one (1D), two (2D) and three (3D) dimensions, present characteristic frequency domains in the dispersion 
curves where light can propagate (bulk bands) and frequency domains where light cannot propagate (gaps). These structures present unusual properties which can be exploited in the control and the guidance of light $[3,4]$. In their 1D version, PCs are well known as optical multilayers of alternating dielectric materials [5]. Such structures exhibit multiple reflections and destructive interference giving rise to forbidden bands where light cannot propagate. This effect is used in designing optical devices such as omnidirectional optical mirrors [6-9], FabryPerot filters [5] and thermophotovoltaics [10]. Recently, quasi-one-dimensional photonic crystals based on coaxial BNC connectors have been used to reproduce several effects related to periodic dielectric media in the radio-frequency region such as: band-gap structures with or without defect modes [11-13], superluminal effects [13, 14], field localization [12], FabryPerot resonators [15] and non-linear effects [16]. Coaxial PCs are more attractive for this purpose than their optical counterparts since they require relatively simple equipment. In addition, the propagation in these structures is monomode [17] and one can obtain very accurate experimental results that may be fitted with a simple 1D theoretical model.

Photonic quasi-crystals are intermediate between periodic and disordered systems [18]. Since the introduction of the Fibonacci dielectric multilayers by Kohmoto et al [19], the propagation of electromagnetic waves in quasi-periodic systems has been the subject of several theoretical and experimental investigations [20-32]. These structures may present several applications such as optical microcavities [31], omnidirectional reflection [32], multiwavelength narrow band optical filters and wavelength division multiplexing systems [26]. Also, it was demonstrated [25] that these systems can provide an interesting alternative to regular photonic crystals for the realization of photonic devices, such as optical filters with a self-similar spectrum and a high wavelength selectivity in the band edge region. Because of their non-interacting nature, electromagnetic waves provide an excellent tool for probing the localization phenomena in comparison with other excitations such as electronic waves. Recently [33], we have studied the propagation and localization of electromagnetic waves in a quasi-periodic coaxial photonic crystal made of loops and segments arranged to a Fibonacci sequence (FS) $S_{k+1}=S_{k} S_{k-1}$ with the initial conditions $S_{1}=A, S_{2}=B$, where $k$ is the generation number. For example $S_{3}=A B A, S_{4}=A B A A B, S_{5}=$ ABAABABAB,$\ldots$ The number of blocks in each generation $k$ is $F_{k}=F_{k-1}+F_{k-2}$ with $F_{1}=1$ and $F_{2}=2$. In [33], we have considered the transmission through a given sequence of the FS inserted between two standard coaxial cables and deduced several properties of the wave propagation in such structures such as the dispersion curves, the phase times and therefore the density of states as well as the group velocities. Each of the two blocks A and B constituting the Fibonacci structure is composed of a loop connected to a segment. When the loop is symmetric (i.e., composed of two identical waveguides), it becomes equivalent to a segment characterized by half the impedance of its constituents. Therefore, each block may be considered as a bi-segment of different impedances. When the segments are standard coaxial cables of $50 \Omega$ impedance, each block becomes a $25 \Omega / 50 \Omega$ bisegment. Our aim was to reproduce Fibonacci structures similar to those used in the pioneering work of Merlin et al [34] on layered media, where each block is composed of two different layers (bilayer). However, because of the length of these structures and the attenuation in the cables, we were not able to check experimentally some peculiar features of FS such as the scaling behaviour of the amplitude and the phase of the transmission coefficient. In this work, we consider a simpler Fibonacci structure where block A is formed by a coaxial cable of length $d_{\mathrm{A}}=1 \mathrm{~m}$ and impedance $Z_{\mathrm{A}}=50 \Omega$ and block B is a symmetric loop of impedance $Z_{\mathrm{B}}=25 \Omega$ and length $d_{\mathrm{B}}=1 \mathrm{~m}$ (see figures 1 (a) and (b)). This structure is equivalent to the one used in our recent theoretical work on acoustic waves in Fibonacci phononic circuits [35] and the works of Kohmoto et al [19, 20] on layered optical media. Our aim in this paper is twofold. 
Block A

(a)

$\mathrm{d}_{1}$

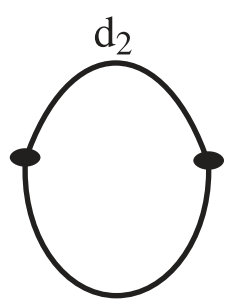

$\mathrm{d}_{3}$ (b)

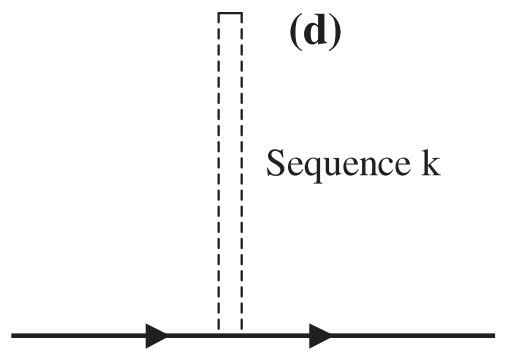

(c)

Sequence k
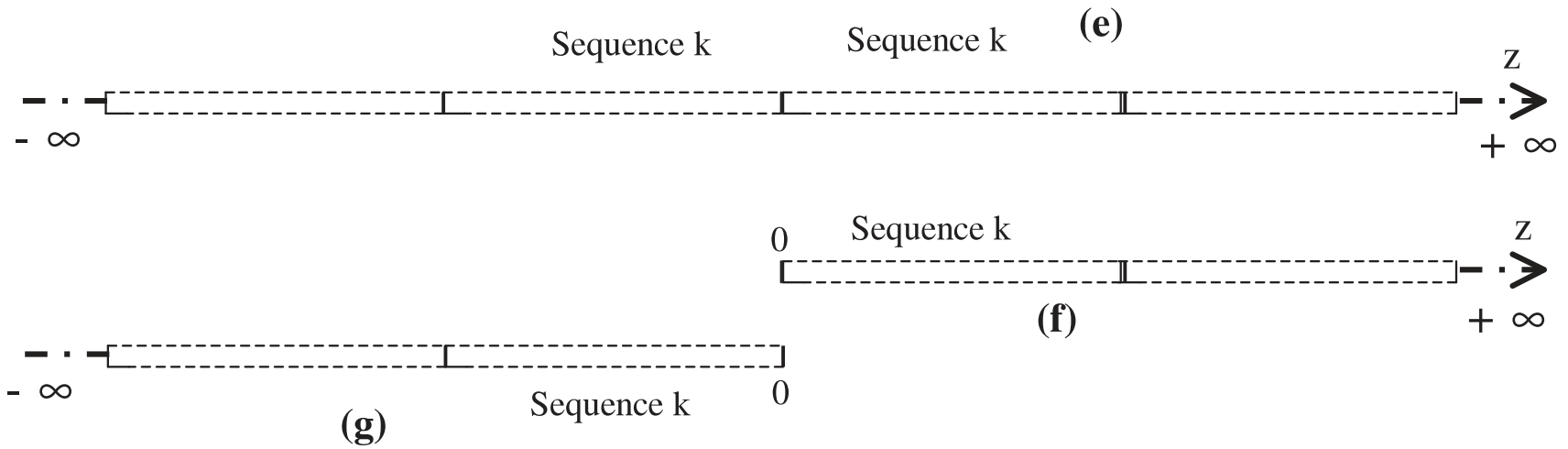

(f)

Figure 1. Schematic representation of blocks $A$ and B constituting the Fibonacci structure. Block A (a) is a simple coaxial cable of length $d_{1}$ and impedance $Z_{1}$. Block B (b) is a loop made of two cables of lengths $d_{2}$ and $d_{3}$ and impedances $Z_{2}$ and $Z_{3}$ respectively. (c) A finite Fibonacci structure inserted horizontally between two waveguides. (d) The same as (c) but for a structure grafted vertically along a guide. (e) A superlattice made of a periodic repetition of a given Fibonacci sequence. (f), (g) Two semi-infinite superlattices obtained by the cleavage of an infinite superlattice (e) between two successive sequences.

(i) In addition to our recent work [33, 35], we give not only theoretical but also experimental evidence of the scaling behaviour of the amplitude and the phase of the transmission coefficient through a finite FS inserted between two semi-infinite waveguides.

(ii) We study a vertical structure formed by a given FS grafted along a guide. This structure enables us to deduce all the eigenmodes of the FS (assuming the vanishing of the magnetic field at the boundaries of the FS).

Different effects related to these quasi-periodic media are observed, including localization and scaling properties.

\section{Method of theoretical and numerical calculation}

\subsection{Interface response theory of continuous media}

Our theoretical analysis is performed with the help of the interface response theory of continuous media, which allows calculation of the Green's function of any composite material. 
In what follows, we present the basic concept and the fundamental equations of this theory [36]. Let us consider any composite material contained in its space of definition $D$ and formed out of $N$ different homogeneous pieces located in their domains $D_{i}$. Each piece is bounded by an interface $M_{i}$, adjacent in general to $j(1 \leqslant j \leqslant J)$ other pieces through subinterface domains $M_{i j}$. The ensemble of all these interface spaces $M_{i}$ will be called the interface space $M$ of the composite material. The elements of the Green's function $g(D D)$ of any composite material can be obtained from [36]

$$
\begin{aligned}
& g(D D)=G(D D)-G(D M) G^{-1}(M M) G(M D) \\
& \quad+G(D M) G^{-1}(M M) g(M M) G^{-1}(M M) G(M D),
\end{aligned}
$$

where $G(D D)$ is the reference Green's function formed out of truncated pieces in $D_{i}$ of the bulk Green's functions of the infinite continuous media and $g(M M)$, the interface element of the Green's function of the composite system. The knowledge of the inverse of $g(M M)$ is sufficient to calculate the interface states of a composite system through the relation [36]

$$
\operatorname{det}\left[g^{-1}(M M)\right]=0 .
$$

Moreover if $U(D)$ represents an eigenvector of the reference system, equation (1) enables the calculation of the eigenvectors $u(D)$ of the composite material and

$$
\begin{aligned}
u(D)=U(D) & -U(M) G^{-1}(M M) G(M D) \\
& +U(M) G^{-1}(M M) g(M M) G^{-1}(M M) G(M D) .
\end{aligned}
$$

In equation (3), $U(D), U(M)$, and $u(D)$ are row vectors. Equation (3) provides a description of all the waves reflected and transmitted by the interfaces, as well as the reflection and transmission coefficients of the composite system. In this case, $U(D)$ is a bulk wave launched in one homogeneous piece of the composite material [17].

\subsection{Inverse surface Green's functions of the elementary constituents}

We consider an infinite homogeneous isotropic dielectric wire $i$ characterized by its characteristic impedance $Z_{i}$. The Fourier transformed Green's function between two points $x$ and $x^{\prime}$ of this wire is

$$
G_{i}\left(x, x^{\prime}\right)=\frac{1}{2} \mathrm{j} Z_{i} \mathrm{e}^{-\alpha_{i}\left|x-x^{\prime}\right|}
$$

where

$$
\alpha_{i}=-\mathrm{j} \frac{\omega}{c} \sqrt{\varepsilon_{i}},
$$

$\varepsilon_{i}$ is the relative permittivity, $\omega$ the angular frequency of the wave, $c$ the speed of light in vacuum, and $\mathrm{j}=\sqrt{-1}$. Before addressing the problem of the FS, it is helpful to know the surface elements of its elementary constituents (see figures 1(a) and 1(b)), namely, the Green's function of a finite segment of length $d_{1}$ and impedance $Z_{1}$, of a loop $(2,3)$ made of two wires 2 and 3 of lengths $d_{2}$ and $d_{3}$ and impedances $Z_{2}$ and $Z_{3}$ respectively, and of a semi-infinite wire $s$ of impedance $Z_{s}$. The finite segment is bounded by two free surfaces located at $x=-d_{i} / 2$ and $x=+d_{i} / 2$. These surface elements can be written in the form of a $(2 \times 2)$ matrix $g_{i}(M M)$, within the interface space $M_{i}=\left\{-d_{i} / 2,+d_{i} / 2\right\}$. The inverse of this matrix takes the form [17]

$$
\left[g_{1}(M M)\right]^{-1}=\left(\begin{array}{cc}
\frac{C_{1}}{Z_{1} \mathrm{~S}_{1}} & -\frac{1}{Z_{1} \mathrm{~S}_{1}} \\
-\frac{1}{Z_{1} \mathrm{~S}_{1}} & \frac{C_{1}}{Z_{1} \mathrm{~S}_{1}}
\end{array}\right),
$$


for the segment (figure 1(a)) and

$$
\left[g_{2,3}(M M)\right]^{-1}=\left(\begin{array}{cc}
\frac{C_{2}}{Z_{2} \mathrm{~S}_{2}}+\frac{C_{3}}{Z_{3} \mathrm{~S}_{3}} & -\frac{1}{Z_{2} \mathrm{~S}_{2}}-\frac{1}{Z_{3} \mathrm{~S}_{3}} \\
-\frac{1}{Z_{2} \mathrm{~S}_{2}}-\frac{1}{Z_{3} \mathrm{~S}_{3}} & \frac{C_{2}}{Z_{2} \mathrm{~S}_{2}}+\frac{C_{3}}{Z_{3} \mathrm{~S}_{3}}
\end{array}\right),
$$

for the loop (figure 1(b)). $C_{i}=\cos \left(\omega d_{i} \sqrt{\varepsilon_{i}} / c\right), \mathrm{S}_{i}=\sin \left(\omega d_{i} \sqrt{\varepsilon_{i}} / c\right)$ and $Z_{i}$ is the impedance of the different wires $(i=1,2,3)$. The inverse of the surface element of a semi-infinite waveguide $s$ characterized by its impedance $Z_{s}$ is given by

$$
\left[g_{s}(0,0)\right]^{-1}=-\frac{\mathrm{j}}{Z_{s}} \text {. }
$$

From equation (7) one can deduce that a symmetric loop made of identical wires of lengths $d_{2}=d_{3}$ and impedances $Z_{2}=Z_{3}$ is equivalent to a single segment of length $d_{2}$ and characterized by the impedance $Z_{2} / 2$. Therefore, block B (figure 1(b)) becomes equivalent to a segment of length $d_{\mathrm{B}}=d_{2}$ and impedance $Z_{\mathrm{B}}=Z_{2} / 2$. We shall also call $d_{\mathrm{A}}=d_{1}$ and $Z_{\mathrm{A}}=Z_{1}$ the length and the impedance of the segment constituting block A respectively. The experimental evidence of the existence of band gaps and defect modes in 1D periodic photonic systems constructed by two alternative coaxial cables of different characteristic impedances was presented recently $[11,12,14,15]$. However, the advantage of the symmetric loop structure lies in the fact that it is not necessary to have two cables of different natures to realize the contrast between the two constituent media of each block. This property could be of practical interest in optical waveguide structures.

\subsection{Transmission coefficient through horizontal and vertical sequences}

The 1D FS can be considered as a finite number of blocks A and B pasted together according to the Fibonacci sequence. The interface domain is made of all the connection points between finite segments and loops. Within the total interface space of the finite sequence, the inverse of the matrix giving all the interface elements of the Green's function $g$ is a finite tridiagonal matrix formed by linear superposition of the elements $\left[g_{i}(M M)\right]^{-1}$ (equations (6) and (7)). The explicit expression of the Green's function elements on the two surfaces bounding the FS can be written as [37]

$$
[g(M M)]^{-1}=\left(\begin{array}{ll}
a & b \\
b & c
\end{array}\right) .
$$

The four matrix elements are real quantity functions of the different parameters of the constituent's elements $g_{i}(M M)$ (equations (6) and (7)). From equations (2) and (9), one can deduce the expression giving the eigenmodes of the FS with (i) vanishing magnetic field boundary conditions, namely

$$
a c-b^{2}=0,
$$

and (ii) vanishing magnetic field on one side and electric field on the other side, namely

$$
c=0 \text {. }
$$

Now, if the finite composite system is sandwiched (grafted) horizontally (vertically) between two homogeneous waveguides labelled $s$ (see figures 1(c) and (d)), then an incident plane wave launched from the left waveguide gives rise to the transmission functions in the right waveguide as

$$
t_{\mathrm{h}}=\frac{2 \mathrm{j} b / Z_{s}}{a c-b^{2}-\left(1 / Z_{s}\right)^{2}-\mathrm{j}(a+c) / Z_{s}},
$$


and

$$
t_{\mathrm{v}}=\frac{-2 \mathrm{j} c / Z_{s}}{a c-b^{2}-2 \mathrm{j} c / Z_{s}},
$$

respectively, where $\mathrm{h}$ and $\mathrm{v}$ stand for horizontal and vertical. The transmission function can be written in an explicit complex form as $t=\alpha+\mathrm{j} \beta=|t| \mathrm{e}^{\mathrm{j} \varphi}$ where $|t|$ is the transmission coefficient, $\varphi=\arctan (\beta / \alpha) \pm m \pi$ is the phase associated with the transmission field and $m$ is an integer. The first derivative of $\varphi$ with respect to the frequency is related to the delay time taken by the wave to traverse the structure. This quantity, called the phase time, is defined by [38]

$$
\tau_{\varphi}=\frac{\mathrm{d} \varphi}{\mathrm{d} \omega}
$$

\subsection{Dispersion relations of infinite and semi-infinite periodic sequences}

The Green's function of the infinite superlattice (SL) made of a periodic repetition of a given sequence (figure 1(e)) is obtained by a linear juxtaposition of the $2 \times 2$ matrices (equation (9)) at the different interfaces. We obtain a tridiagonal matrix. After a Fourier transform, we obtain the following expression giving the dispersion relation of an infinite SL [39]:

$$
\cos (K D)=-(a+c) / 2 b,
$$

where $K$ is the propagation vector and $D$ the period of the SL ( $D=\sum_{i=1}^{F_{k}} d_{i}$, where $F_{k}$ is the number of blocks in generation $k$ ). In the same way, the dispersion relation giving the surface modes for a semi-infinite SL lying in the half space $z>0$ (figure 1(f)) is given by

$$
a c-b^{2}=0,
$$

together with the condition

$$
\left|\frac{b}{a}\right|<1 .
$$

The latter condition (equation (17)) ensures that the waves are decaying from the surface when penetrating into the SL. We obtain the same expression (equation (16)) for the semi-infinite SL lying in the half space $z<0$ (figure $1(\mathrm{~g})$ ) but with the condition

$$
\left|\frac{b}{a}\right|>1 \text {. }
$$

This result shows that if a surface mode appears on the surface of one SL, it does not appear on the other surface of the complementary SL. Moreover, equation (16) shows that the expression giving the surface modes for two complementary SLs is exactly the same expression giving the eigenmodes of one sequence (equation (10)).

\section{Numerical and experimental results}

As mentioned above, we consider a Fibonacci structure made of two blocks A and B. The wires constituting these blocks are made of standard coaxial cables (i.e. $Z=50 \Omega$ and $\varepsilon=2.3$ ). Block $\mathrm{A}$ is a coaxial cable of length $d_{\mathrm{A}}=1 \mathrm{~m}$ and impedance $Z=Z_{\mathrm{A}}=50 \Omega$, whereas block B is a loop made of two identical cables of length $d_{\mathrm{B}}=1 \mathrm{~m}$ and impedance $Z=50 \Omega$ (figures 1(a) and (b)). As mentioned before, this loop is equivalent to a segment of length $d_{\mathrm{B}}=1 \mathrm{~m}$ and impedance $Z_{\mathrm{B}}=Z / 2=25 \Omega$. We shall focus on the transmission coefficient when a given Fibonacci sequence is either inserted horizontally between, or grafted vertically to, two semi-infinite waveguides of type A. In particular, we shall show that these two geometric systems may give different information on the propagation and localization of electromagnetic waves in such structures. 


\subsection{The case of the horizontal sequence}

In this subsection, we consider a given Fibonacci sequence inserted horizontally between two semi-infinite waveguides (figure 1(c)). Figures 2(b)-(g) show the transmission coefficient for the generations $\mathrm{S}_{4}$ (five blocks), $\mathrm{S}_{5}$ (eight blocks), $\mathrm{S}_{6}$ (13 blocks), $\mathrm{S}_{7}$ (21 blocks), $\mathrm{S}_{8}$ (34 blocks) and $S_{9}$ (55 blocks), respectively. The solid (dashed) curves represent the theoretical results with (without) absorption, whereas open circles correspond to the experimental ones. The experiments were performed using standard coaxial cables assembled together with metallic T-shaped connectors. The cross section of the cables being negligible compared to their length and to the propagation wavelength, the assumption of monomode propagation is then satisfied. The transmission measurements have been realized by using the tracking generator coupled to a spectrum analyser in the frequency range of $10-100 \mathrm{MHz}$. The attenuation inside the coaxial cables was simulated by introducing a complex dielectric constant $\varepsilon\left(\varepsilon=\varepsilon^{\prime}-\mathrm{j} \varepsilon^{\prime \prime}\right)$. The attenuation coefficient $\alpha^{\prime \prime}$ can be expressed as $\alpha^{\prime \prime}=\varepsilon^{\prime \prime} \omega / c$. On the other hand, the attenuation specification data supplied by the manufacturer of the coaxial cables in the frequency range of $10-100 \mathrm{MHz}$ can be approximately fitted with the expression $\ln \left(\alpha^{\prime \prime}\right)=\gamma+\delta \ln (\omega)$, where $\gamma$ and $\delta$ are two constants. From this fitting procedure, a useful expression for $\varepsilon^{\prime \prime}$ as a function of frequency can be obtained under the form $\varepsilon^{\prime \prime}=0.017 f^{-0.5}$ where the frequency $f$ is expressed in hertz. The experimental results are very well fitted by the 1D model using the Green's function method. One can notice in figure 2 that the attenuation inside the cables induces transmission depletion, especially at high frequencies.

Two regions of frequencies may be distinguished in figures $2(\mathrm{~b})-(\mathrm{g})$ : the regions where the transmission falls down rapidly to zero as the generation number increases, which correspond to the forbidden modes (transmission gaps), and the regions where the transmission is more noticeable, which correspond to the allowed modes (transmission bands). These results clearly show the existence of more than one gap (transmission dips) in comparison with the transmission spectrum of the periodic case (figure 2(a)), especially for high generations (figure $2(\mathrm{~g})$ ). This is the consequence of the band-gap fragmentation in quasi-periodic structures. An interesting result in figures $2(\mathrm{~b})-(\mathrm{g})$ is the existence of new features around the central gap frequency $f_{\mathrm{c}} \simeq 49.34 \mathrm{MHz}$ of the periodic structure; these resonances present a certain recursive order which is a characteristic of Fibonacci systems. This property, called the scaling relation $[19,20]$, has been interpreted as a sign of localization of the waves in Fibonacci systems. Kohmoto et al [19] have shown that the scaling behaviour of the transmission coefficient is characterized by the scaling factor $[19,20]$

$$
F=\sqrt{1+4(1+I)^{2}}+2(1+I),
$$

where $I$ is an invariant which remains constant at every step of the recursive procedure. This invariant is given by [19]

$$
I=\frac{1}{4}\left(\frac{Z_{\mathrm{B}}}{Z_{\mathrm{A}}}-\frac{Z_{\mathrm{A}}}{Z_{\mathrm{B}}}\right)^{2} \sin ^{2}(\omega d \sqrt{\varepsilon} / c)
$$

where $Z_{\mathrm{A}}=50 \Omega$ and $Z_{\mathrm{B}}=25 \Omega$ are the impedances of the cables and loops respectively. Also, it has been demonstrated $[19,20]$ that one can expect scaling around $\phi=\omega d \sqrt{\varepsilon} / c=$ $(2 m+1) \pi / 2$, where the quasiperiodicity is most effective ( $m$ is an integer). This implies that the transmission coefficient should exhibit a self-similar behaviour around the central frequency $f_{\mathrm{c}}=(2 m+1) 49.34 \mathrm{MHz}$ with $T_{j+3}=T_{j}$ (the period of the transmission coefficient is three recursions) with a scaling factor $F$. For this frequency, equations (19) and (20) lead to $I=$ 0.526 and thus $F=6.4061$. This is clearly illustrated in the insets of figures $2(\mathrm{~b})-(\mathrm{g})$ near $f_{\mathrm{c}}$. Note the scale change of the frequency axis in the insets of figures 2(b), (c), and (d) as compared to figures 2(e), (f) and (g) respectively. We remark that $S_{4}\left(S_{7}\right), S_{5}\left(S_{8}\right)$ and $S_{6}\left(S_{9}\right)$ resemble 

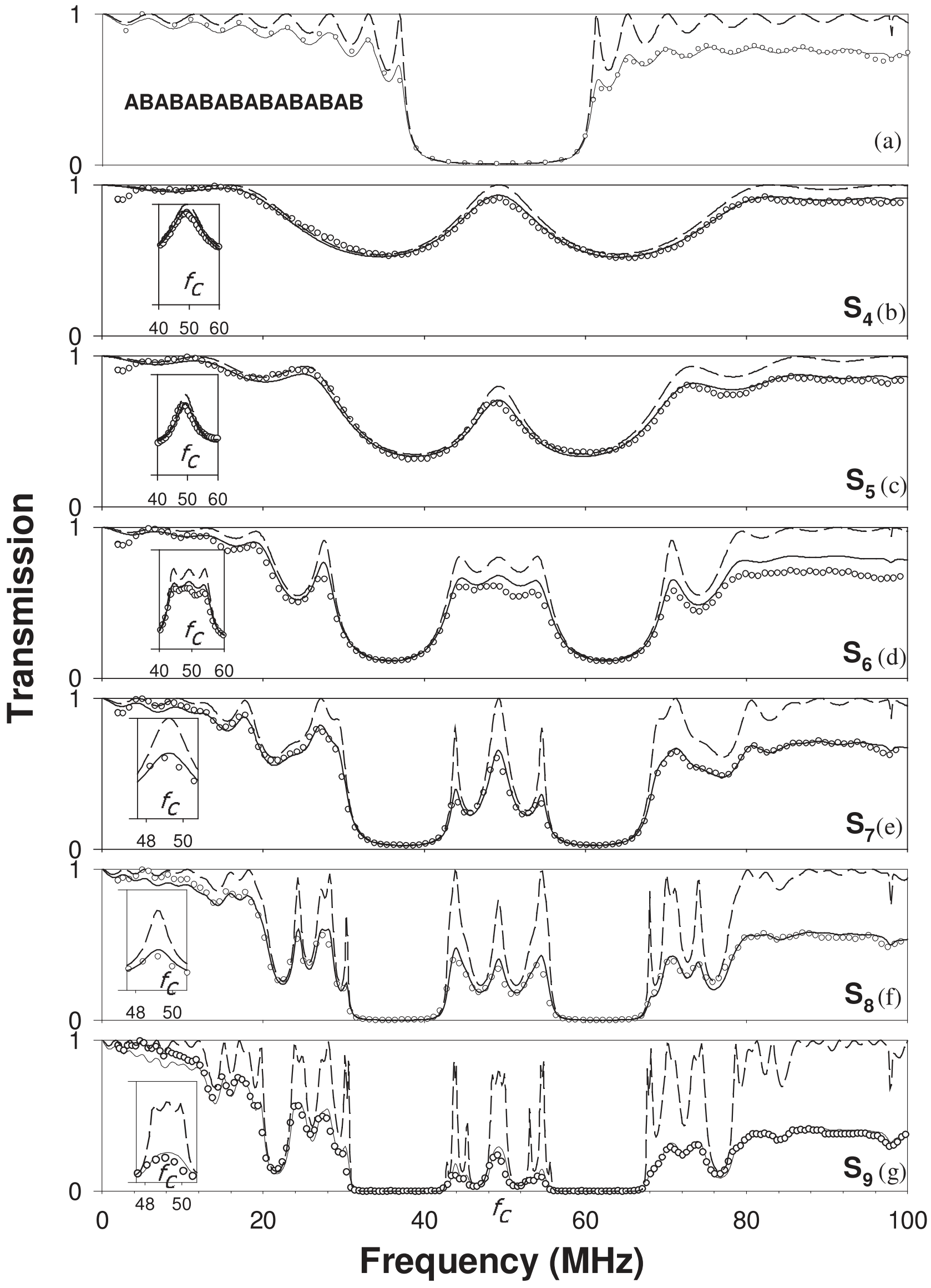

Figure 2. Theoretical (solid curves) and experimental (open circles) variations of the transmission coefficient as a function of the frequency for different structures. Block $\mathrm{A}$ is a cable of length $d_{1}=1 \mathrm{~m}$ and impedance $Z_{1}=50 \Omega$, while block B is a symmetric loop with $d_{2}=d_{3}=1 \mathrm{~m}$ and $Z_{2}=Z_{3}=50 \Omega$. (a) The periodic structure; (b) $-(\mathrm{g})$ generation $S_{k}(k=4-9)$ of the Fibonacci structure. The dashed curves in (a) $-(\mathrm{g})$ are the theoretical curves when the absorption is not taken into account. Blocks A and B in the periodic and Fibonacci structures are the same as in figure 1. Note the scale change of the frequency axis near the central frequency $f_{\mathrm{c}}$ in the insets of the figures associated with $\mathrm{S}_{4}, \mathrm{~S}_{5}$ and $\mathrm{S}_{6}$ as compared to $\mathrm{S}_{7}, \mathrm{~S}_{8}$ and $\mathrm{S}_{9}$ respectively. 
each other near $f_{\mathrm{c}}$ with a periodicity of three and a scaling factor $F$ as was found by Gellermann et al [20] in optical layered media. One can notice, however, that due to the dissipation in the cables the similarities between the different curves around $f_{\mathrm{c}}$ do not show exactly the same features as they should in the absence of dissipation (see the dashed curves). This is in particular the case for high generations (see the insets of figures 2(d) and (g)). However, as will be shown below, this self-similarity is better illustrated in the transmission phase time.

It is well established that outside the Fibonacci band gaps the waves are critically localized [39]. In contrast with the fully disordered (Anderson) localized case, these critically localized modes display weaker decay than exponential, most likely by a power law, and have a rich self-similar structure [40]. In order to understand the spatial localization of the different modes in figure 2, we plotted in figure 3 the local density of states ${ }^{4}$ (LDOS) as a function of the space position $z$ for the modes lying at $f=98.68,49.34$ and $68.08 \mathrm{MHz}$ (i.e. for the reduced frequencies $\phi=\pi, 0.5 \pi$ and $0.69 \pi$ ) in figure 2(f) (eighth generation). The LDOS reflects the square modulus of the electric field inside the structure. These modes could be classified respectively as the following. (i) Extended modes as shown in figure 3(a) for the completely transparent mode $(\phi=\pi)$, for which the transmission is unity [39]. The wavefunction, namely the electric field distribution, follows the structure of the Fibonacci sequence (see the inset of figure 3(a)). Similar results are found for electronic and electromagnetic waves in layered media [41, 42]. (ii) Self-similar modes as shown in figure 3(b) for the mode lying at $\phi=0.5 \pi$. The corresponding LDOS shows a self-similar behaviour $[43,44]$ around the main peak every three generations (see the inset of figure 3(b) displayed for the 11th generation). (iii) Bandedge modes as shown in figure 3(c) for the mode $\phi=0.69 \pi$. The LDOS shows a noticeable similarity to the band edge resonances occurring in the periodic structure (see the inset of figure 3(c)) but is less regular. Band edge resonances in photonic periodic crystals are shown not to be localized states since their extension scales linearly with the system size and they do not decay to zero $[43,45]$. In contrast, the Fibonacci band edge resonances may decay via a power law due to an optical path gradient occurring during the growth of the Fibonacci samples [25, 46]. As pointed out in [47], these resonances could serve as a new type of complex cavity in 1D multilayer structures that provides the feedback for laser action in the field of random laser systems.

Another important quantity that characterizes the interaction of the incident photon with the different modes in the FS is the transmission phase time $\tau_{\varphi}$. This quantity is interpreted as the time needed for a photon to complete the transmission process. The phase time in symmetric loop structures is equivalent to the total density of states [13, 37]. Figure 4(a) gives the phase time as a function of the frequency for the periodic structure as in figure 2(a). Similarly to the density of states in 1D structures, the phase time exhibits large values near the band edges and small oscillations inside the allowed bands (which are indicative of the discrete modes in the finite size structure) and vanishes inside the forbidden bands. In the frequency domain of the forbidden bands of the periodic structure around $f_{\mathrm{c}}$, the phase time associated with the FS (figures 4(b)-(g)) also shows a self-similarity (three recursions). In addition, because of the small effect of the attenuation in the cables on the phase, the self-similarity around $f_{\mathrm{c}}$ is better illustrated in the spectra of the phase time $\left(\mathrm{S}_{4}\left(\mathrm{~S}_{7}\right), \mathrm{S}_{5}\left(\mathrm{~S}_{8}\right)\right.$ and $\left.\mathrm{S}_{6}\left(\mathrm{~S}_{9}\right)\right)$ than in the spectra of the amplitude (see the insets of figure 2). To our knowledge, until now there has been no experimental study on the phase time scaling behaviour in FS. It is worth mentioning that these results are different from those we obtained recently [33] when blocks A and B were composed of a bisegment instead of one segment. In this case, the periodicity is of order six instead of three and therefore the scaling factor is about $F^{2}$ instead of $F$.

4 The detail of calculations of LDOS is given in [51]. 

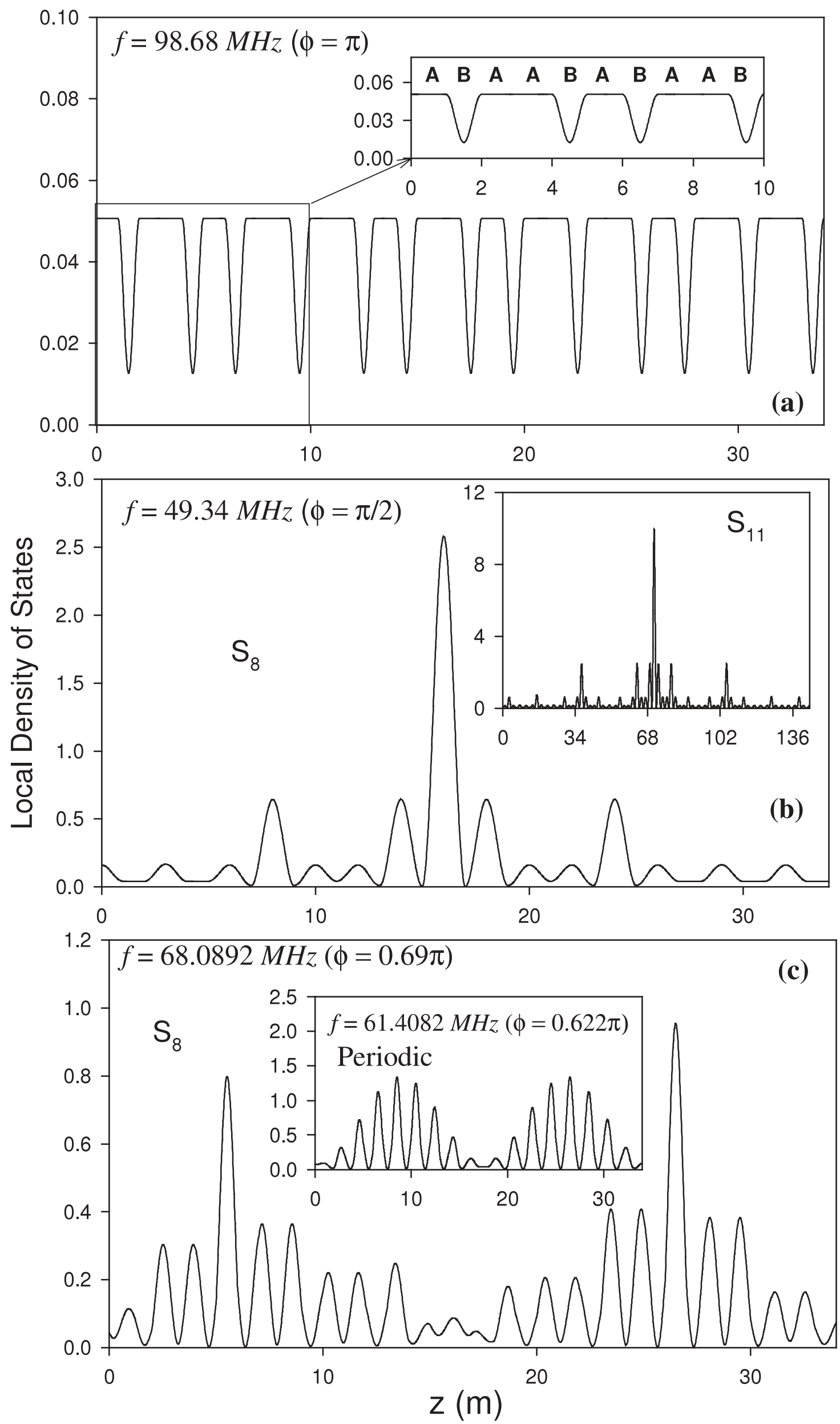

Figure 3. The local density of states LDOS (in arbitrary units) as a function of the space position $z$ for three frequencies from figure 2(f): (a) $\phi=\pi$ (middle of the band), (b) $\phi=0.5 \pi$ (central frequency) and (c) $\phi=0.69 \pi$ (band edge). The inset of (b) corresponds to the LDOS associated with the 11th generation at $\phi=0.5 \pi$. The inset of (c) corresponds to the LDOS associated with the periodic structure at $\phi=0.622 \pi$. 


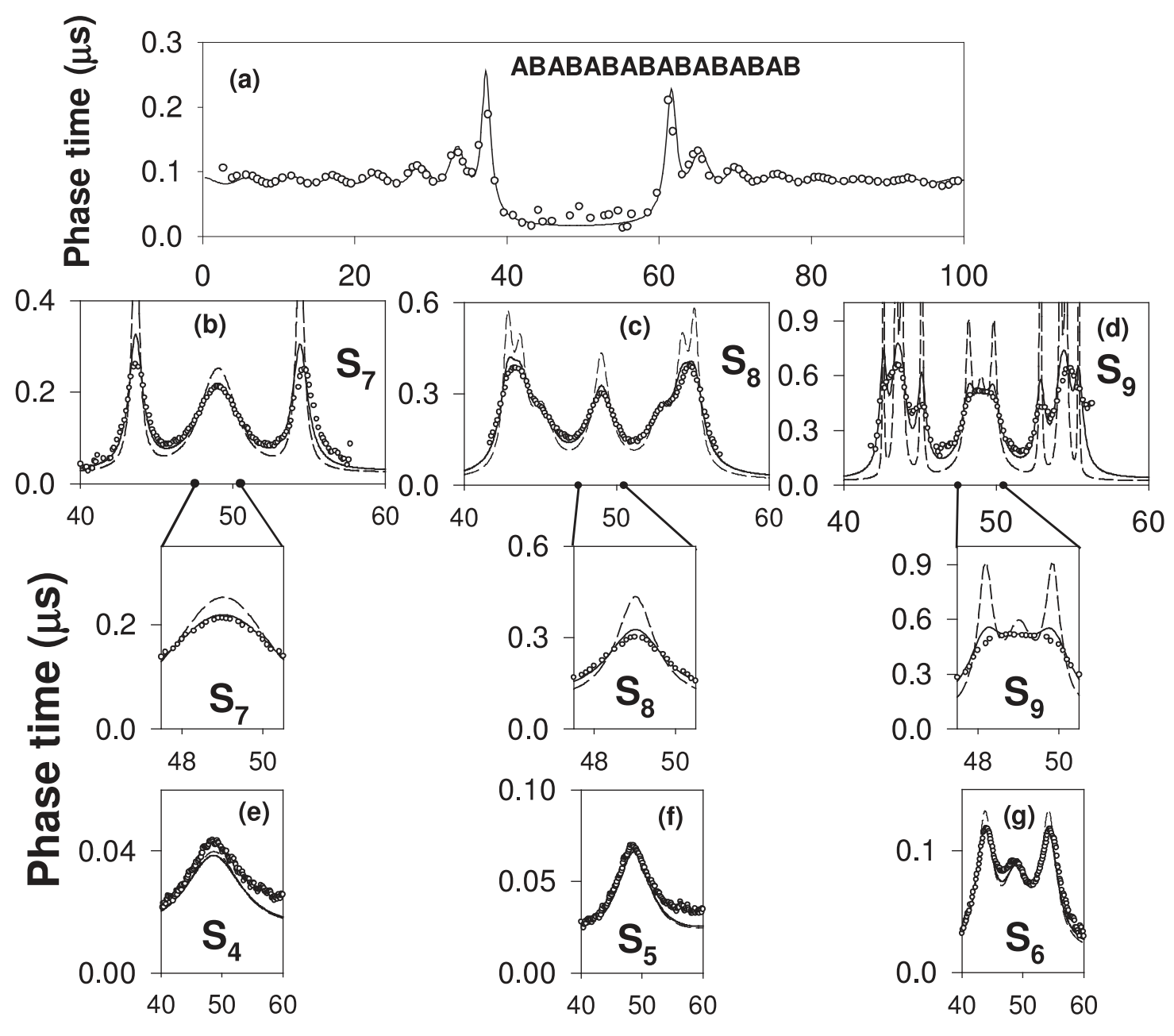

Frequency $(\mathrm{MHz})$

Figure 4. (a) The same as figure 2(a) but for the phase time. (b)-(g) The same as figures 2(b)-(g) but for the phase time in the frequency domain 40-60 MHz.

From the phase time one can deduce the group velocity defined as $v_{\mathrm{g}}=L / \tau_{\varphi}[48]$ where $L$ is the total length of the structure, i.e. the sum of the lengths of the A and B blocks constituting the structure (here $L=F_{k}$ metres). In the case of a periodic structure (figure 5(a)), an anomalous dispersion occurs inside the gaps and velocities greater than the speed of light are expected $[13,14]$. Inside the passbands, the group velocity is equal to $0.66 c$, which is the normal speed of wave propagation in the cables used in these experiments. Near the band edge a strongly reduced group velocity associated with large delay times can be observed. In the case of an FS (figures 5(b)-(g)), which induces large phase times in the frequency domain 40$60 \mathrm{MHz}$ (figures 4(b)-(g)), small group velocities $v_{\mathrm{g}} \simeq 0.3 \mathrm{c}$ lower than the normal speed in the cables (i.e. 0.66c) can be observed. This value is the same as the one found by Munday and Robertson [49] in a periodic structure made of two alternating different coaxial cables with an embedded defect cable. Therefore, the FS may be used as a tool to reduce the propagation speed of waves in such structures.

\subsection{The case of the vertical sequence}

Now, we consider the second structure, where the FS is attached vertically to two waveguides (figure 1(d)). From the comparison of equations (10) and (13) one can see that the maxima of the transmission (i.e. $t_{\mathrm{v}}=1$ ) occur at the eigenfrequencies of the finite FS with vanishing magnetic field on both ends. However, the comparison of equations (11) and (13) shows that 

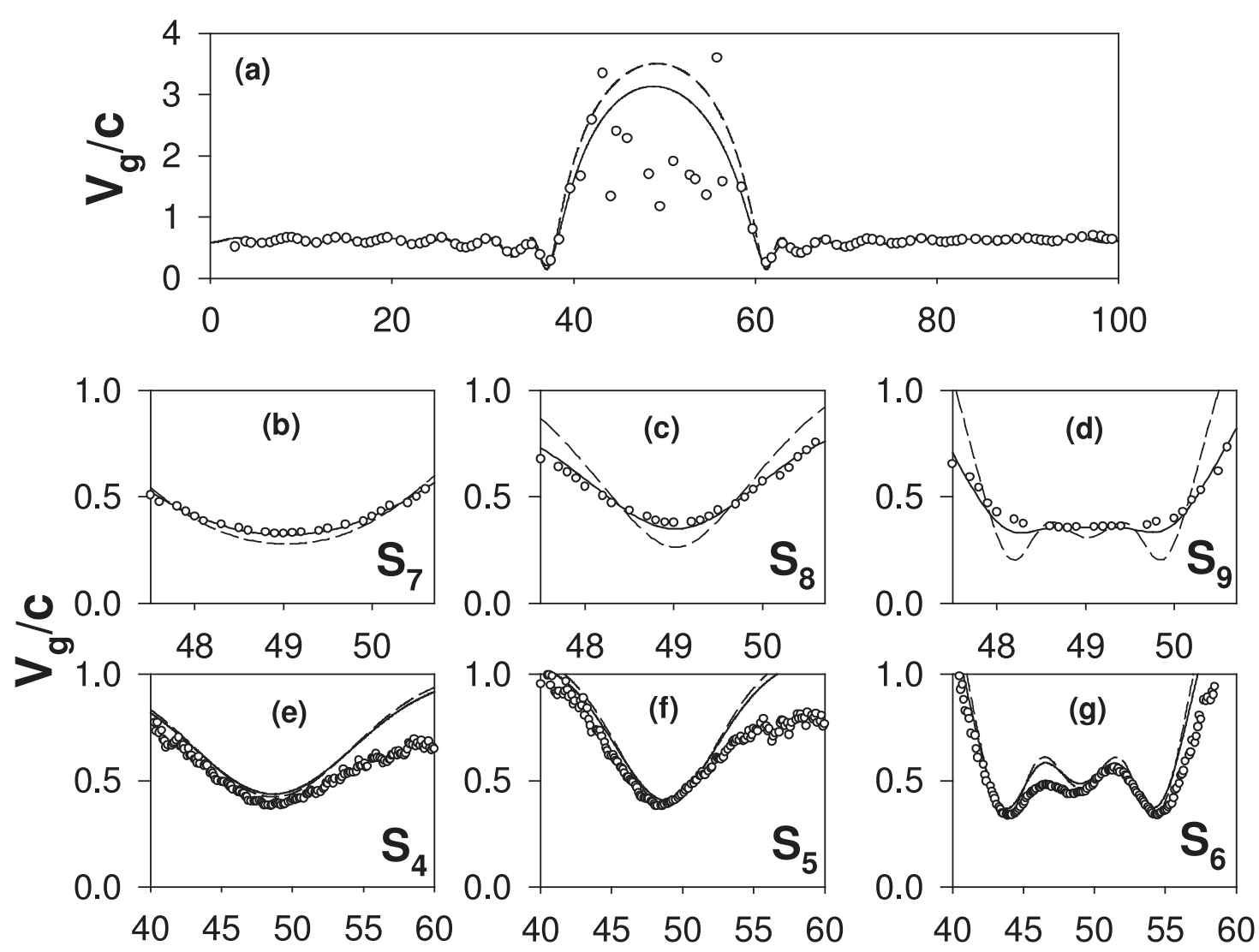

Frequency $(\mathrm{MHz})$

Figure 5. (a) The same as figure 4 but for the group velocity.

the minima of the transmission (i.e. $t_{\mathrm{v}}=0$ ) occur at the eigenfrequencies of the finite FS with vanishing magnetic field on one side and electric field on the other side. Transmission spectra for the generations $\mathrm{S}_{3}-\mathrm{S}_{8}$ are presented in figure 6. The theoretical results (full curves) are in good agreement with experimental results (open circles). In figure 7 we have reported as dots the frequencies corresponding to the maxima of the spectra in figure 6 for different generations. One can notice that, as predicted, the number of eigenmodes of the FS increases with increasing generation number. Another interesting point is the accumulation of these modes in certain frequency regions and their absence from other regions that coincide with dips in the transmission spectra of figure 2.

In general, the spatial localizations of the eigenmodes of the FS with vanishing magnetic fields on both ends present different behaviours in comparison with those of the FS inserted between two waveguides. An example of the dependence of the LDOS on the media surrounding the structure is sketched in figure 8 for the mode lying exactly at the central frequency $f_{\mathrm{c}}=49.34 \mathrm{MHz}$. One can notice that the localization of the electric field goes from the middle of the structure to its extremities when the impedance of the surrounding media decreases from $Z_{s}=Z_{\mathrm{A}}$ to $Z_{s}=0$. In particular, one can notice (figure 9(a)) that, contrary to the results of figure $3(\mathrm{~b})$, the whole spectra of the LDOS associated with the modes lying at $f_{\mathrm{c}}=49.34 \mathrm{MHz}$ (see figure $7(\mathrm{~b})$ which corresponds to figure 7 (a) enlarged in the frequency domain $40-60 \mathrm{MHz}$ ) are not self-similar as illustrated in the LDOS of $\mathrm{S}_{11}$ as compared to $S_{8}$ sketched in the inset of figure $9(\mathrm{~b})$. However, a magnification of the spectra associated with $S_{11}$ in the space domain $z \leqslant 34 \mathrm{~m}$ gives rise to similar spectra as those of $S_{8}$ and $S_{5}$. This is shown in figures $9(b)$ and (c), where we have magnified the curve of $S_{11}$ (figure 8(a)) and compared it to the curves of $S_{8}$ and $S_{5}$ sketched in the insets of figures 9 (b) and (c) respectively. For the modes lying at an almost constant frequency ( $f=41.93 \mathrm{MHz}$ ) 


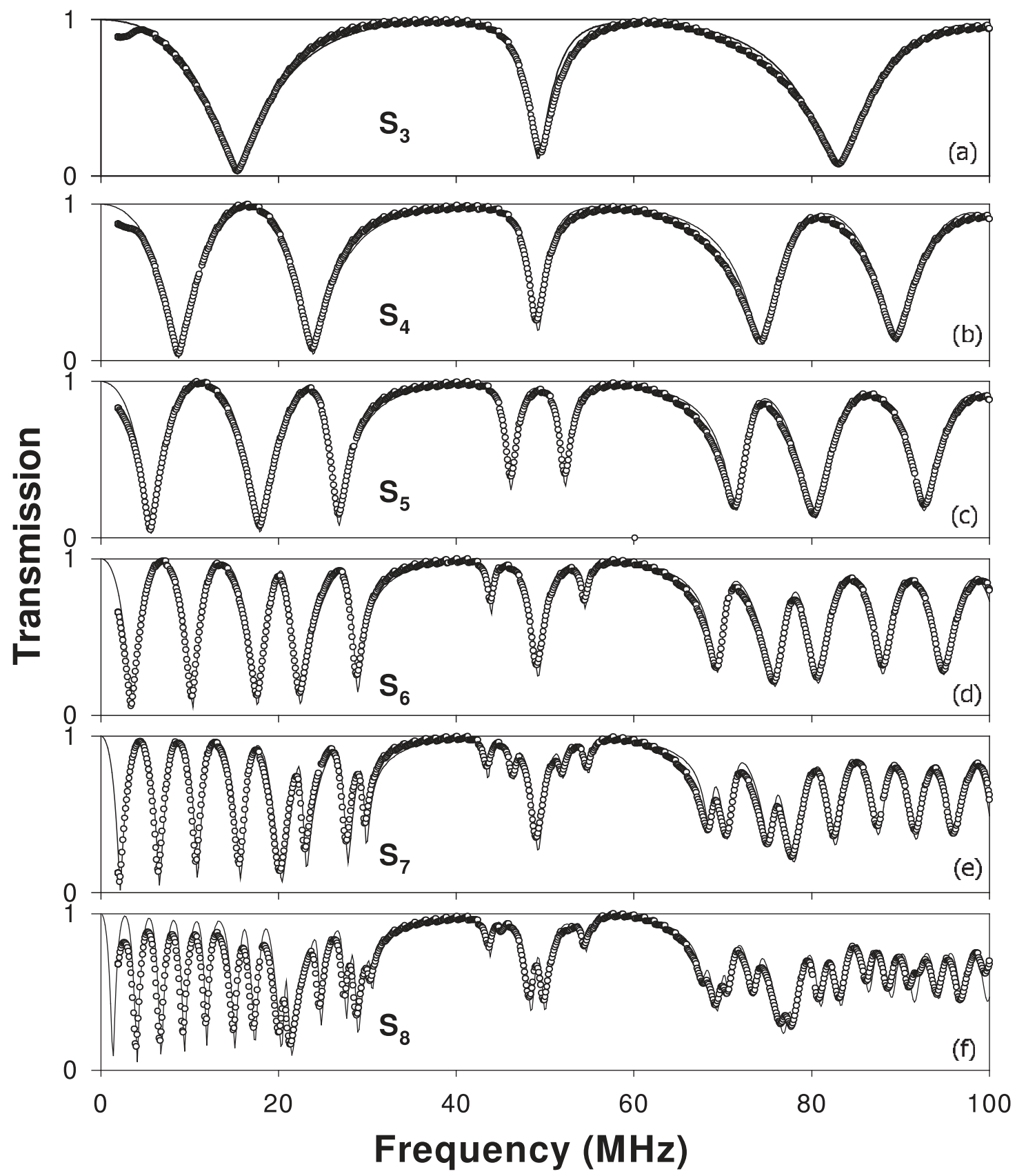

Figure 6. Theoretical (solid curves) and experimental (open circles) variations of the transmission coefficient as a function of the frequency for a finite Fibonacci sequence grafted vertically along a guide (figure 1(d)) for generations $\mathrm{S}_{3}-\mathrm{S}_{8}$.

until the eighth generation (figure 7(b)), these modes are induced by one of the two surfaces bounding the FS as shown in figure 9(d) for the modes labelled 1 and 2 in figure 7(b). We observe now a decrease of the LDOS from the surface when penetrating into the structure with almost the same localization. This is also the case for the modes lying at $f=57.01 \mathrm{MHz}$ (figure 7(b)). These modes are without analogue in the case of an FS surrounded by media of different impedances. For the modes lying at $f=98.68 \mathrm{MHz}$ (i.e. $\phi=\pi$ ), one can notice (dashed curve in figure 9(e)) that these modes show regular oscillations (extended modes). This behaviour is different from the one obtained for the same mode but in the horizontal structure, where the LDOS follows a Fibonacci line shape (see figure 3(a)). Indeed, an analysis of the LDOS similar to the one sketched in figure 8 shows that the LDOS follows a Fibonacci line shape for different impedances of the surrounding media until $Z_{s}=0$, where this mode shows regular oscillations (figure 9(e)). Concerning the other modes of the FS, they exhibit an irregular shape inside the structure (see the full curve in figure 9(e) associated with the mode labelled 3 in figure 7(b)). These modes could be qualified as critical modes. It is worth to notice 


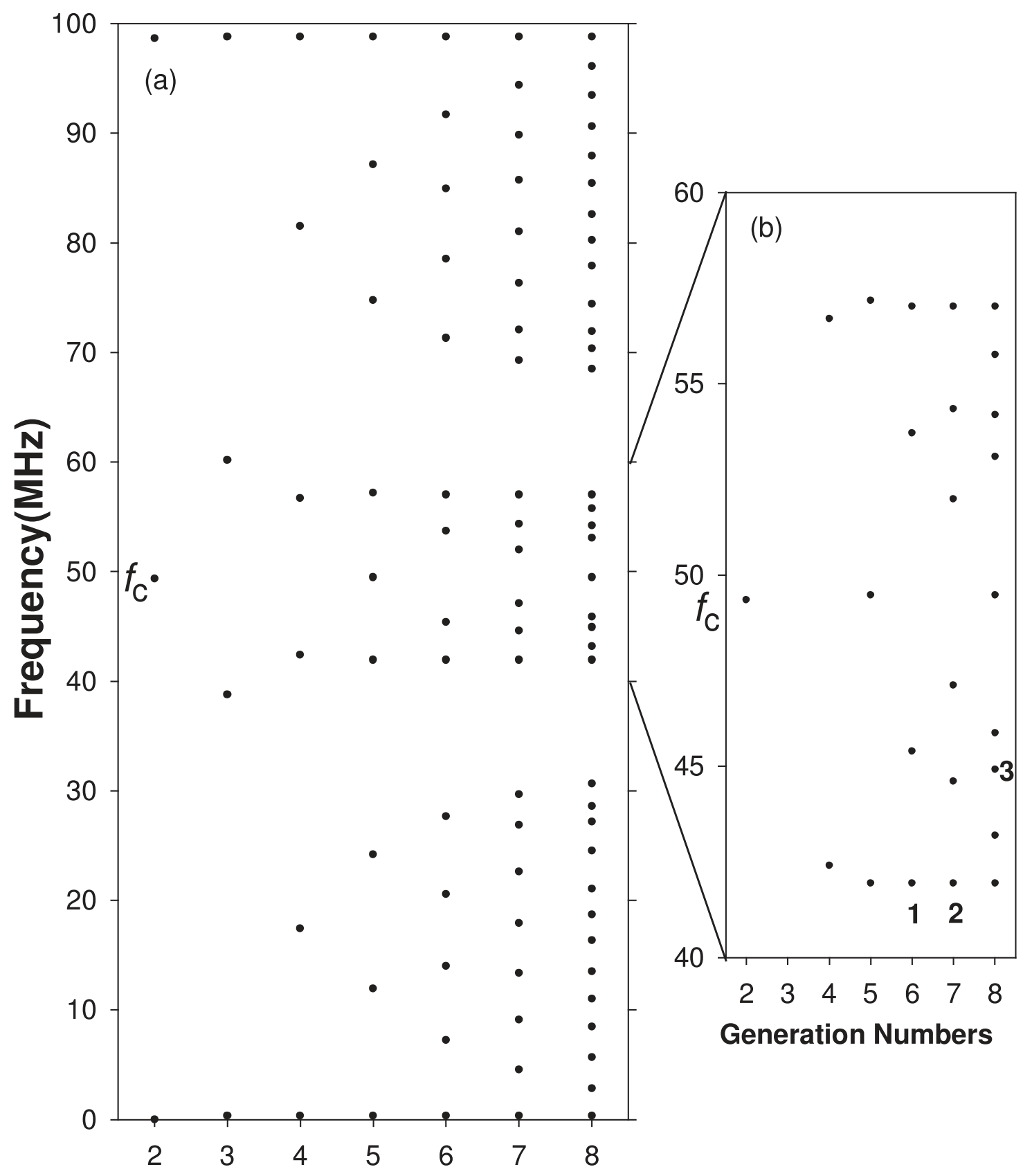

Generation Numbers

Figure 7. (a) Eigenfrequencies (dots) of a finite FS with vanishing magnetic field on both sides as a function of the generation numbers. The eigenmodes are obtained from the maxima of the transmission spectra of figure 6. (b) The same as (a) but enlarged in the frequency domain 40$60 \mathrm{MHz}$.

that similar results to those presented here are found theoretically for other excitations in the FS such as plasmon polaritons and magnetostatic excitations [50]. As mentioned above, the minima of the transmission spectra of figure 6 give the eigenmodes of the FS with vanishing magnetic field on one side and electric field on the other side. These results are reported in figure 10. One can notice that the eigenmodes of such a structure are quite different from those of the FS with vanishing magnetic field on both ends (figure 7(a)).

As mentioned in section 2.4, the eigenfrequencies of the finite FS with vanishing magnetic field on both ends coincide with the surface modes of two complementary superlattices obtained by the cleavage of an infinite superlattice made of a periodic repetition of a given sequence. To confirm these results, we have plotted in figure 11 the distribution of the bandwidths (heavy lines) of an infinite superlattice. These bulk bands are separated by gaps where surface modes for the semi-infinite superlattice lying at $z>0$ (figure $1(\mathrm{f}))(z<0$, figure $1(\mathrm{~g}))$ are given by 


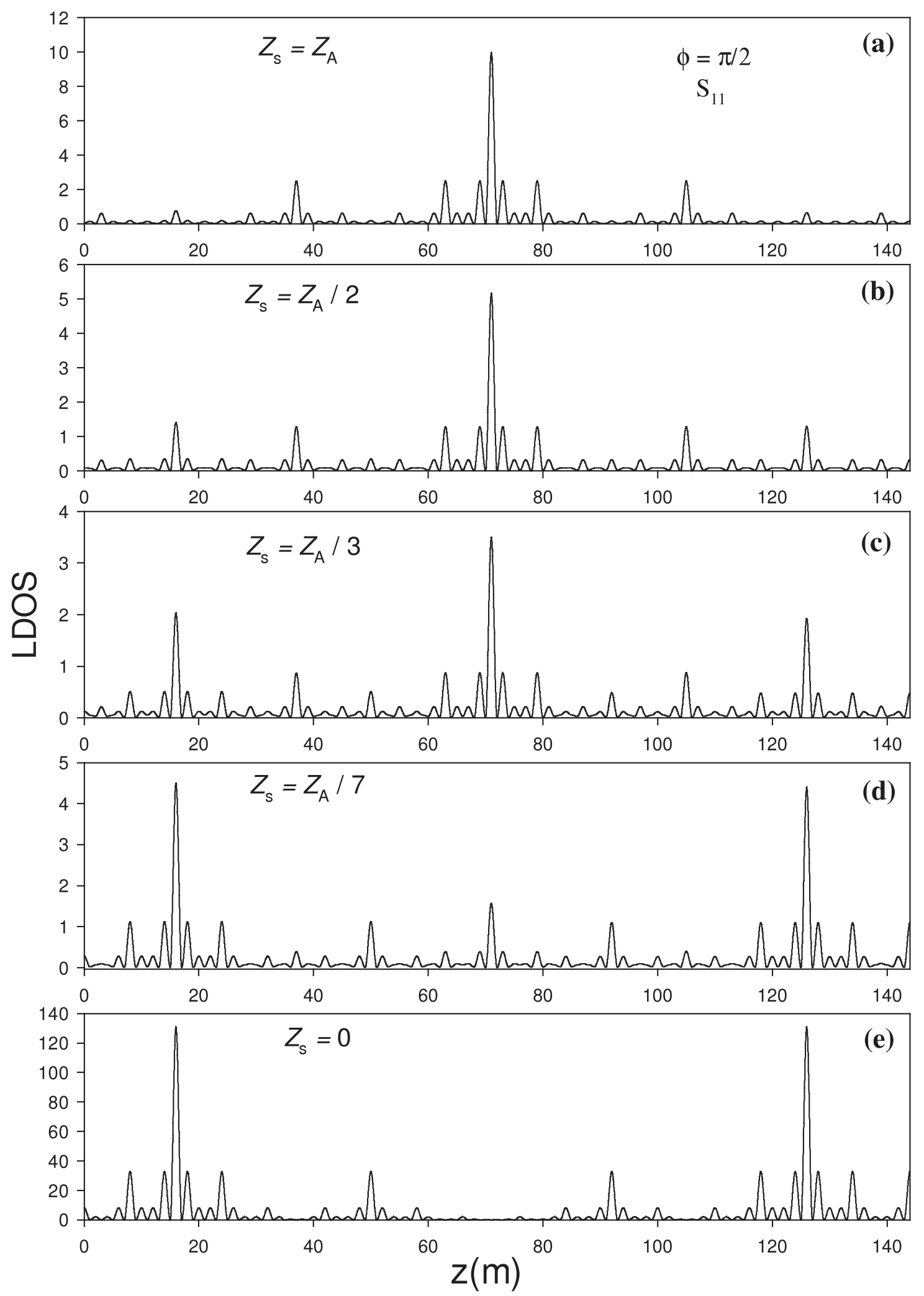

Figure 8. The LDOS (in arbitrary units) as a function of the space position $z$ for generation $S_{11}$ at the central frequency $f_{\mathrm{c}}=49.34 \mathrm{MHz}$ and different impedances of the surrounding media: (a) $Z_{s}=Z_{\mathrm{A}}$, (b) $Z_{s}=Z_{\mathrm{A}} / 2$, (c) $Z_{s}=Z_{\mathrm{A}} / 3$, (d) $Z_{s}=Z_{\mathrm{A}} / 7$ and (e) $Z_{s}=0$.

open circles (filled triangles). One can notice that, apart from the modes lying around 0 and $100 \mathrm{MHz}$, all the eigenmodes of one finite FS (figure 7(a)) coincide with the surface modes of two complementary superlattices (figure 11). In addition, we obtain a generalization of a rule obtained before for transverse elastic waves in $N$-layer superlattices [51]; namely, there exists one surface mode per gap for each generation when we consider two complementary semiinfinite superlattices together. As the number of bands is equal to the number of blocks $F_{k}$ for each generation $k$ in the frequency domain $\phi<\pi$ (i.e. $f<100 \mathrm{MHz}$ ), the number of gaps 

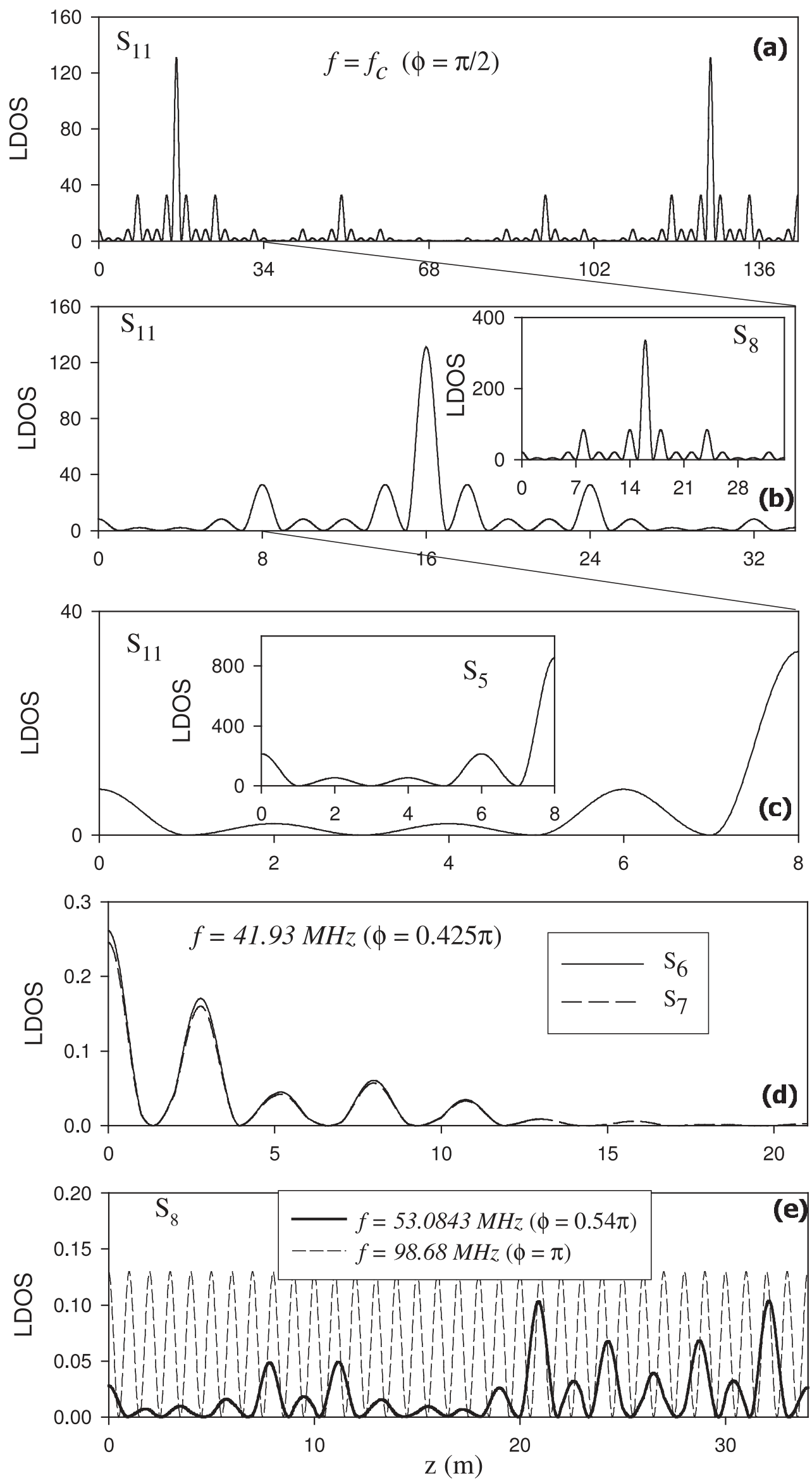

Figure 9. The LDOS (in arbitrary units) as a function of the space position $z$ for two frequencies. (i) $f=f_{\mathrm{c}}$ and different generations: (a) $\mathrm{S}_{11}$, (b) $\mathrm{S}_{11}$ enlarged in the space position $z<34 \mathrm{~m}$ (the inset represents the LDOS for generation $\mathrm{S}_{8}$ ) and (c) $\mathrm{S}_{11}$ enlarged in the space position $z<8 \mathrm{~m}$ (the inset represents the LDOS for generation $S_{5}$ ). Notice the high similarity between the enlarged magnification of the results for $\mathrm{S}_{11}$ and the results for $\mathrm{S}_{8}$ and $\mathrm{S}_{5}$. (ii) $f=41.93 \mathrm{MHz}$; the LDOSs for the modes labelled 1 and 2 in figure 7(b) for generations $S_{6}$ and $S_{7}$ are sketched in figure 8(d) by solid and dashed lines respectively. (e) LDOS for the mode labelled 3 (full curve) in figure 7(b); the dashed curve corresponds to the mode lying at $\phi=\pi(f=98.68 \mathrm{MHz})$. 


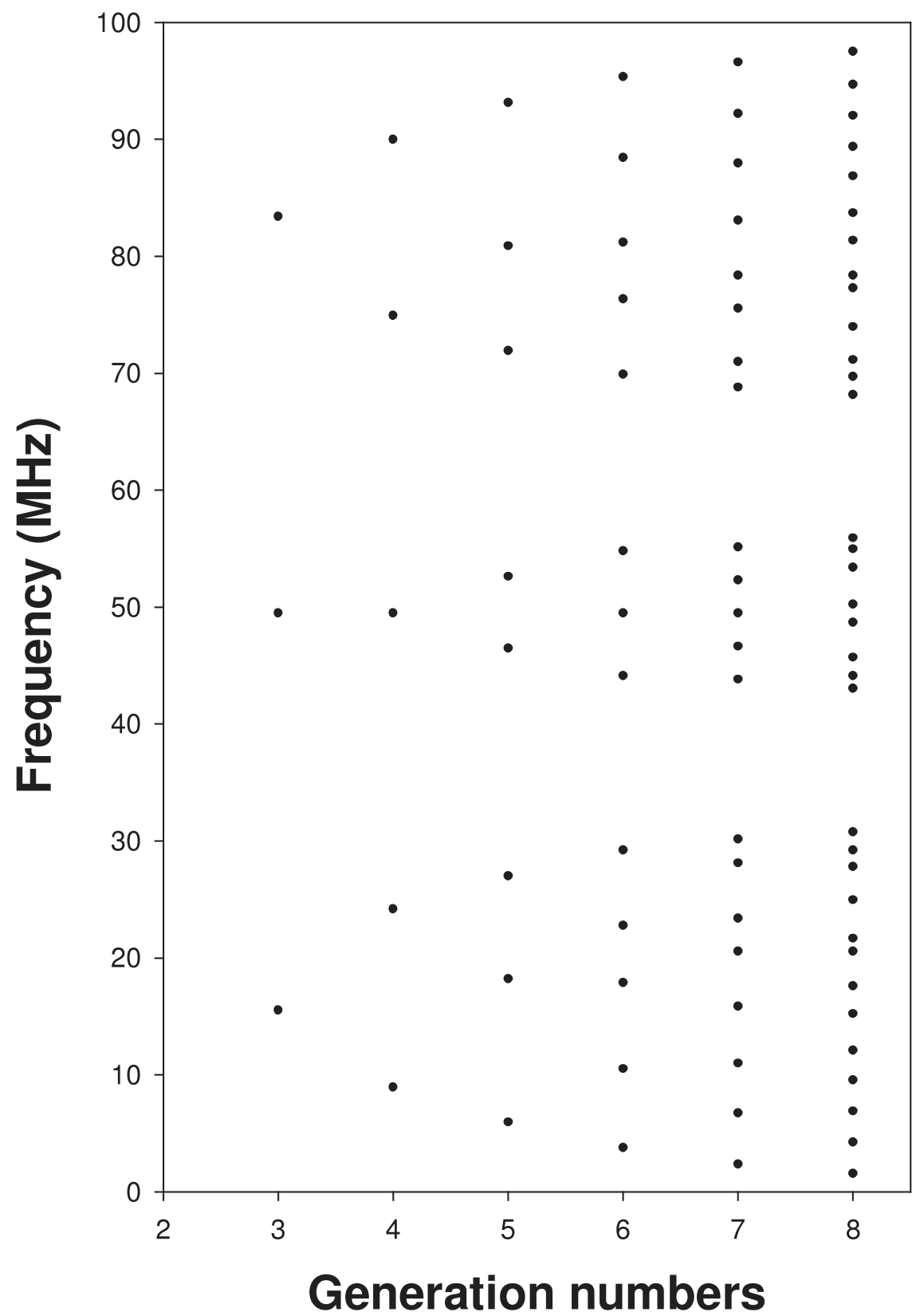

Figure 10. (a) Eigenfrequencies (dots) of a finite FS with vanishing magnetic field on one side and electric field on the other side as a function of the generation numbers. The eigenmodes are obtained from the minima of the transmission spectra of figure 6.

and therefore of surface modes is equal to $F_{k}-1$. A detailed analysis of the bulk and surface modes of infinite, finite and semi-infinite periodic FSs including the scaling and multifractal behaviours has been presented elsewhere [52].

\section{Summary and conclusions}

In this paper, we have presented theoretical and experimental results of propagation and localization of electromagnetic waves in Fibonacci structures made of coaxial cables. The two blocks constituting the system are respectively a simple coaxial cable and a symmetric loop that play the role of a segment with half the impedance of its constituting wires. We have considered the propagation of electromagnetic waves through such structures in two different ways, where each way gives interesting results on the modes of the FS.

(i) By inserting the structure horizontally between two waveguides, we were able to reproduce different results known in layered media as the splitting of the bands and the selfsimilarity between the transmission spectra with a scaling factor when the generation 


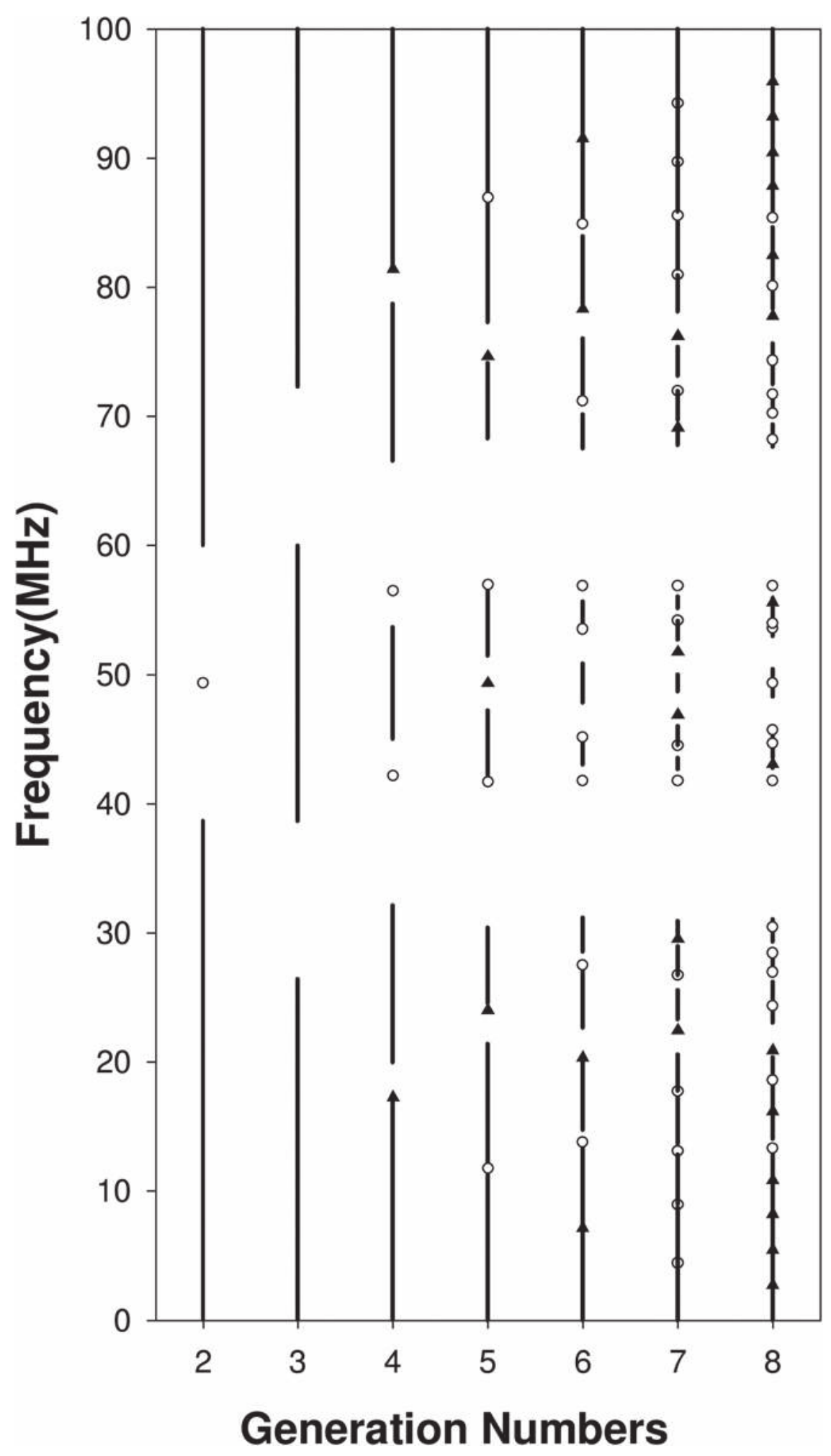

Figure 11. Distribution of the bandwidth (vertical lines) of a periodic superlattice (figure 1(e)) as a function of the generation number. Open circles and filled triangles correspond to the surface modes of two semi-infinite complementary superlattices lying at $z>0$ (figure 1 (f)) and $z<0$ (figure 1(g)) respectively. Notice the similarity between the positions of these modes and those of figure 7(a).

number increases. In addition, we have shown that besides the transmission amplitude, an analysis of the phase time enables us to determine the density of states as well as the group velocities in these structures.

(ii) By grafting the structure vertically, we have shown that the maxima of the transmission coefficient give the eigenmodes of the FS with vanishing magnetic field at the boundaries, whereas the minima of the transmission coefficient give the eigenmodes of the FS with vanishing magnetic field on one side and electric field on the other side. 
The spatial localizations of the modes of the FS are very sensitive to the different boundary conditions on both ends. Some modes of the FS with vanishing magnetic field on both ends fall at an almost constant frequency as a function of the generation number. These modes may exhibit either a strong localization at the surface terminations of the FS, a self-similar behaviour of order three or just an oscillating behaviour inside the FS.

\section{Acknowledgments}

EE and YE gratefully acknowledge the hospitality of Institut d'Électronique, de Microélectronique et de Nanotechnologie (IEMN) and UFR de Physique, Université de Lille 1. This work was supported by le Fond Européen de Développement Régional (FEDER), INTERREG III France-Wallonie-Flandre (PREMIO) and le Conseil Régional Nord-Pas de Calais.

\section{References}

[1] Yablonovitch E 1987 Phys. Rev. Lett. 582059

[2] John S 1987 Phys. Rev. Lett. 582486

[3] See, for example Joannopoulos J D, Meade R D and Winn J N 1995 Photonic Crystals (Princeton, NJ: Princeton University Press)

[4] Soukoulis C M (ed) 1993 Photonic Band Gap and Localization (New York: Plenum)

Soukoulis C M (ed) 1996 Photonic Band Gap Materials (Dordrecht: Kluwer)

[5] Yeh P 1988 Optical Waves in Layered Media (New York: Wiley)

[6] Fink Y, Winn J N, Fan S, Chen C, Michel J, Joannopoulos J D and Thomas E L 1998 Science 2821679

[7] Dowling J P 1998 Science 2821841

[8] Yablonovitch E 1998 Opt. Lett. 231648

[9] Mir A, Akjouj A, El Boudouti E H, Djafari-Rouhani B and Dobrzynski L 2001 Vacuum 63197

[10] Celanovic I, O'Sullivan F, Ilak M, Kassakian J and Perrault D 2004 Opt. Lett. 29863

O’Sullivan F, Celanovic I, Jovanovic N, Kassakian J, Akiyama S and Wada K 2005 J. Appl. Phys. 97033529

[11] Schneider G J, Hanna S, Davis J L and Watson G H 2001 J. Appl. Phys. 902642

[12] Haché A and Slimani A 2004 Am. J. Phys. 72916

[13] El Boudouti E H, Fettouhi N, Akjouj A, Djafari-Rouhani B, Mir A, Vasseur J, Dobrzynski L and Zammouri J 2004 J. Appl. Phys. 951102

[14] Haché A and Poirier L 2002 Phys. Rev. E 65036608

Munday J N and Robertson W 2002 Appl. Phys. Lett. 80518

[15] del Mar Sanchez-Lopez M, Davis J A and Crabtree K 2003 Am. J. Phys. 711314

[16] Poirier L and Haché A 2001 Appl. Phys. Lett. 782626

[17] Vasseur J, Akjouj A, Djafari-Rouhani B, Dobrzynski L and El Boudouti E H 2004 Surf. Sci. Rep. 541

[18] Albuquerque E L and Cottam M G 2003 Phys. Rep. 376225

Albuquerque E L and Cottam M G 2004 Polaritons in Periodic and Quasiperiodic Structures (Amsterdam: Elsevier)

[19] Kohmoto M, Sutherland B and Iguchi K 1987 Phys. Rev. Lett. 582436

[20] Gellermann W, Kohmoto M, Sutherland B and Taylor P C 1994 Phys. Rev. Lett. 72633

[21] Hattori T, Tsurumachi N, Kawato S and Nakatsuka H 1994 Phys. Rev. B 50 R4220

[22] Pelster R, Gasparian V and Nimtz G 1997 Phys. Rev. E 557645

[23] Chow L and Guenther K H 1993 J. Opt. Soc. Am. A 102231

[24] Peng R W, Liu Y M, Huang X Q, Qin F, Wang M, Hu A, Jiang S S, Feng D, Ouyang L Z and Zou J 2004 Phys. Rev. B 69165109

[25] Ghulinyan M, Oton C J, Dal Negro L, Pavesi L, Sapienza R, Colocci M and Wiersma D S 2005 Phys. Rev. B 71094204

[26] Peng R W, Wang M, Hu A, Jiang S S, Jin G J and Feng D 1998 Phys. Rev. B 571544

[27] Sibilia C, Nefedov I S, Scolara M and Berlotti M 1998 J. Opt. Soc. Am. B 151947

[28] Huang X, Wang Y and Gong C 1999 J. Phys.: Condens. Matter 117645

[29] Liu N H 1997 Phys. Rev. B 553543

[30] Lavrinenko A V, Zhukovsky S V, Sandmirski K S and Gaponenko S V 2002 Phys. Rev. E 65036621 
[31] Macia E 2001 Phys. Rev. B 63205421

[32] Lusk D, Abdulhalim I and Placido F 2001 Opt. Commun. 198273

[33] Aynaou H, El Boudouti E H, El Hassouani Y, Akjouj A, Djafari-Rouhani B, Vasseur J, Benomar A and Velasco V R 2005 Phys. Rev. E 72056601

[34] Merlin R, Bajema K and Clarke R 1985 Phys. Rev. Lett. 551768

Bajema K and Merlin R 1987 Phys. Rev. B 364555

[35] Aynaou H, El Boudouti E H, Djafari-Rouhani B, Akjouj A and Velasco V R 2005 J. Phys.: Condens. Matter 174245

[36] Dobrzynski L 1991 Surf. Sci. Rep. 11139

[37] Lahlaouti M L H, Akjouj A, Djafari-Rouhani B, Dobrzynski L, Hammouchi M, El Boudouti E H, Nougaoui A and Kharbouch B 2001 Phys. Rev. B 63035312

[38] Buttiker M and Landauer R 1982 Phys. Rev. Lett. 491739

Hauge E H and Stoveng J A 1989 Rev. Mod. Phys. 61917

[39] Macia E and Dominguez-Adame F 1996 Phys. Rev. Lett. 762957

[40] Fijiwara T, Kohmoto M and Tokihiro T 1989 Phys. Rev. B 407413

Soukoulis C M and Economou E N 1982 Phys. Rev. Lett. 481043

[41] Ryu C S, Oh G Y and Lee M H 1992 Phys. Rev. B 465162

Ryu C S, Oh G Y and Lee M H 1993 Phys. Rev. B 48132

[42] Vasconcelos M S, Albuquerque E L and Mariz A M 1998 J. Phys.: Condens. Matter 105839

[43] Kohmoto M, Sutherland B and Tang C 1987 Phys. Rev. B 351020

Kohmoto M and Banvar J R 1986 Phys. Rev. B 34563

[44] Jin D and Jin G 2005 Phys. Rev. B 71014212

[45] Scalora M, Flynn R J, Reinhardt S B, Fork R L, Bloemer M J, Tocci M D, Bowden C M, Ledbetter H S, Bendickson J M and Dowling J P 1996 Phys. Rev. E 54 R1078

[46] Dal Negro L, Oton C J, Gaburro Z, Pavesi L, Johnson P, Lagendijk A, Righini R, Colocci M and Wiersma D S 2003 Phys. Rev. Lett. 90055501

[47] Wiersma D, Sapienza R, Mujumdar S, Colocci M, Ghulinyan M and Pavesi L 2005 J. Opt. A: Pure Appl. Opt. $7 \mathrm{~S} 190$

[48] Zhu J N, Liu N, Zheng H and Chen H 2000 Opt. Commun. B 174139

[49] Munday J N and Roberston W M 2003 Appl. Phys. Lett. 831053

[50] Liu N-h, He Y, Feng W-g and Wu X 1995 Phys. Rev. B 5211105

Kang S S 2002 Phys. Rev. B 65064401

[51] El Boudouti E H, Djafari-Rouhani B, Akjouj A and Dobrzynski L 1996 Phys. Rev. B 5414728

[52] El Hassouani Y, Aynaou H, El Boudouti E H, Djafari-Rouhani B, Akjouj A and Velasco V R 2006 Phys. Rev. B 74035314 